\title{
EDITORIAL
}

\section{The Journal of Human Hypertension and the Millennium}

It is not unreasonable that the first issue of the Journal of Human Hypertension for this, the Millennium year, should take time to look back whilst also looking to the future. Some of our readers may be suffering from 'Millennium fatigue' and might point out that life, time and space are a continuum and that the pace of medical research is unlikely to be influenced by any particular date. However, it is helpful to take stock and 01/01/00 seems as good a time as any.

The Journal of Human Hypertension was started in 1987. At the time there was no reasonably efficient clinical journal devoted to high blood pressure and related issues. The existing journals contained many excellent papers but a large proportion were concerned with animal research and were therefore of only passing use to clinicians. This is not in any way to denigrate animal research but merely to argue that many clinicians do not find it particularly relevant to their work. Right from the start we opted to be an international journal, although at first many members of the Editorial Board were British. These members, however, have gradually been replaced as the years have gone by and it has become clear that the journal is a success.

Whilst working on the fourth edition of the BMJ publication of the $A B C$ of Hypertension I was struck by the fact that many things we wrote in the 3rd edition are now untrue! Clinicans who have not had postgraduate training in the field of hypertension and who qualified before 1987 may therefore be seriously out-of-date and may be mismanaging their patients.

It is interesting to look back at the topics that we held to be true in 1987. In those days, the angiotensin-converting enzyme inhibitors were new and exciting and the angiotensin receptor antagonists were almost unheard of. We believed then that diastolic blood pressure was of prime importance and we were uncertain of the significance of systolic pressure. How things have changed! Recently Professor Peter Sever from St Mary's Hospital has even gone as far as suggesting that we should stop measuring diastolic blood pressure altogether!

In the early years of our publication, the salt hypothesis remained highly controversial and some clinicians considered the whole business to be 'utter rubbish'. Again things have changed. The publication of the INTERSALT project and the detailed meta-analyses of the effects of short-term salt restric- tion have more or less ended the controversy (although there are still a few doubters!).

Another major advance has been our totally altered understanding of non-insulin dependent diabetes mellitus. In 1987, diabetic patients were lucky ever to have their blood pressure measured but now we know that the height of their blood pressure is a better prognostic indicator than the height of the blood sugar. Furthermore, the value of antihypertensive therapy, even with a thiazide diuretic, is actually greater in diabetes than in non-diabetes. These findings prompted Professor Hans Ibsen to comment that type II diabetes is a cardiovascular disease and should be looked after by cardiovascular doctors.

Similarly, our attitude towards plasma cholesterol have been transformed. The amazing success of the statin group of drugs, not only in lipid lowering, but also at preventing heart disease and, somewhat surprisingly, strokes, have transformed our whole approach to patients' cardiovascular prevention. Now our patients are looked at from the point of view of their total cardiovascular risk and not just the height of their blood pressure. This change is reflected in the many guidelines that have appeared recently.

Ten years ago there were major anxieties that it was possible to drop blood pressure too low. Many papers suggested that there was a 'J' curve, with a critical level of diastolic blood pressure below which treatment appeared to do more harm than good. This was largely seen in case-control studies and retrospective series. When this problem was subjected to a randomised controlled trial in the HOT study the findings were very reassuring. In particular, patients with pre-existing cardiovascular disease benefited most if their diastolic blood pressure was targeted at $80 \mathrm{~mm} \mathrm{Hg}$ rather than a higher figure.

Turning to drugs, we have witnessed, over the last 15 years, the rise and then gentle fall of the betablockers. Originally these drugs, known to be effective at the secondary prevention of heart disease, were considered candidates to be routine first-line therapy on the grounds that they might bring about primary prevention of coronary heart disease in hypertensive patients. Trial evidence, however, disappointingly showed that this was not the case. The question as to which are the best antihypertensive drugs remains unanswered. Only the thiazides, the beta-blockers and more recently the calcium chan- 
nel blockers have been validated in long-term outcome studies. It is interesting that, having risen and then fallen, the thiazide diuretics are rising again as the optimum first-line drugs in elderly hypertensive patients. The 'anti-thiazide propaganda' to which we have been subjected over the past years has been grossly overstated, largely by the drug companies that make other classes of drugs. It was not until 1991 that we were confident of the correct dose of bendrofluazide and, at this dose, the metabolic sideeffects of thiazides can be regarded as trivial.

In the early 1990s there were a great many papers which suggested that calcium channel blockers caused haemorrhage, suicide, cancer and general mayhem. These were largely based on case-control studies and many observers frankly doubted them. In fact the randomised placebo-controlled Syst-Eur trial showed that calcium channel blockers were in fact very good at preventing heart attacks and strokes with no adverse effects on cancer or cardiovascular death. Furthermore, the calcium blockers were particularly useful in the sub-set of patients with diabetes. The calcium channel blocker scare is over. There is absolutely no substitute for well-conducted randomised controlled trials, either comparing treatment with placebo or comparing different treatments. In the field of hypertension we have been well served, although serious question marks must remain over the ABCD study and the CAPP trial.

The next generation of clinical trials, many currently underway, are addressing the question of how we should reduce blood pressure. With very few exceptions we know who to treat, so now the question is what are the best drugs. The first of these long-term outcome trials was published on the 20th November 1999 in the Lancet. The STOP Hypertension II study group appears to have shown that calcium channel blockers, ACE inhibitors and 'conventional drugs' are roughly equally effective in preventing heart attacks and strokes. Professor Martin Kendall declared it 'a draw'. Again this is reassuring evidence that calcium channel blockers are safe but somewhat disappointing evidence that the ACE inhibitors do not convey as much advantage as they might in theory have done. There remains little doubt the ACE inhibitors are the drugs of choice in diabetic nephropathy and in some cases the non-diabetic nephropathy and they remain the treatment of choice in heart failure. Their superior effects on left ventricular hypertrophy (LVH) however may be related to duration of therapy. Most drugs will regress $\mathrm{LVH}$ but it is possible that the ACE inhibitors do this more quickly. We can now look forward to several more studies of new vs old antihypertensive drugs, including in the American ALLHAT study and the Anglo-Scandinavian Cardiac Outcome Trial (ASCOT).

The arrival in 1995 of the angiotensin receptor antagonists was impressive because it soon became clear that they were almost completely devoid of side-effects. Most clinicians, when faced with a drug which is alleged to be devoid of side-effects, will give a contemptuous snort and make some remark like 'I have heard that before!'. In fact clinical experience bears out the original findings of the remarkable tolerability of losartan and its competitors. There was a brief period when it even appeared that losartan may be better than captopril in the treatment of heart failure although this finding has not been borne out by the expanded ELITE II study. Losartan is probably as good as ACE inhibition in the treatment of heart failure but is much more tolerable.

Research into genes is now fashionable and when it comes to grant-giving bodies that is where the money goes! Anyone with any epidemiological training will know that there will never be a gene for hypertension as such becaue it is such a multifactorial condition. There is, however, a distinct possibility that there may be a gene for left ventricular hypertrophy, renal damage and salt sensitivity and papers in this issue of the journal address these topics.

This review cannot possibly cover all the changing scenes in the hypertension world. We will, however, be publishing a special 'Millennium Issue' of the Journal of Human Hypertension timed to coincide with the International Society of Hypertension meeting in August 2000 in Chicago. This will contain 17 authoritative review articles by internationally recognised experts.

The major explosion of new information means that the guidelines published in 1993 can be regarded as obsolete. The American Joint National Committee (JNC6) published new guidelines in 1997. Wisely, the British Hypertension Society opted to wait until 1999 and publish their guidelines in two versions. The longer, more detailed guidelines were published in this journal ( $\mathrm{Hum}$ Hypertens (1999) 13, 569-592). A shorter version appeared in the British Medical Journal. One must however express some sympathy with clinicians in the field of general (internal) medicine who are subjected to guidelines for almost all medical conditions. How does one keep up? Probably the Cochrane database is the best method of proceeding. Clinicians will, via the Internet, be able to obtain overview analyses of the results of the major trials and can then make decisions based on the latest information. Maybe one day we will be able to link up the Cochrane database (named after the distinguished epidemiologist, Dr Archie Cochrane) with the patients clinical records so that we can see instantly what the database recommends for an individual patient.

Finally, there is the problem of blood pressure measurement where we are now in a state of total anarchy. Mercury is being phased out, probably rightly, not only because it is potentially hazardous but also because mercury manometers are a source of observer bias and error. This is highlighted in a paper from St George's Hospital in this issue of the journal. What, however, are we to replace the mercury with? Only a very small number of automatic or semi-automatic blood pressure machines have passed the criteria for accuracy laid down by the British Hypertension Society or the AAMI. Many papers have been published reporting that various electronic machines are inaccurate. The advantage 
of electronic machines is that one can measure blood pressure effortlessly several times during a consultation. Single casual blood pressure readings measured with an inaccurate machine must be regarded as totally unacceptable. The increasing use of 24-h ambulatory blood pressure monitoring may improve things, if only because it provides a large number of readings away from the clinical environment. However, ambulatory monitoring must be regarded with a certain degree of caution. It is becoming increasingly apparent that white-coat hypertension is not as innocent as was originally thought. It may simply be early hypertension. The British Hypertension Society is probably right to suggest that all patients who have had raised blood pressures that have settled should be rechecked annually, preferably by a well trained hypertension nurse.

The failure of the adequate control of blood pressure in the majority of hypertensive patients has prompted initiatives, by the American Society of Hypertension, to create a speciality of 'hypertensionology'. There is a need for experts in hypertension even though all doctors in every branch of medicine do have to manage high blood pressure. The British Hypertension Society is also starting moves to increase higher training in hypertension for cardiologists, nephrologists and clinical pharmacologists. This initiative will take some years to fall into place but it is interesting in that so far no-one has denied that such action is necessary. At the moment, most physicians receive almost no training in hypertension, one of the commonest chronic medical conditions in the developed and developing nations and a major course of heart attacks and strokes. This cannot go on!

This journal has published a randomised controlled trial of nurse vs doctor management of hypertension and the nurses obtained better results with fewer drop outs. Clearly, if nurses are to play an increasing part in the management of hypertension they will need higher training just as much as the doctors. There is at the moment no formal apparatus to facilitate higher training for nurses in hypertension and cardiovascular disease. It is possible that in the United Kingdom the British Hypertension Society together with the Nurses Hypertension Association may be able to organise formal training for nurses and it is hoped that similar initiatives will take place in other countries.

This brief Cook's tour of the field of hypertension clearly must omit many areas of debate and contention and many areas with new information. It is to be hoped that the pages of this journal will, over the coming years, fill in the gaps. We owe a debt of gratitude not only to our Editorial Board and Coeditors but also to the very large number of very patient and helpful referees. Finally of course, our main debt of gratitude must go to the authors of all the papers which have been submitted to this journal over the years.

May I wish all our readers a happy and glorious New Year, New Century and New Millennium.

Professor DG Beevers Editor-in-Chief University Department of Medicine City Hospital Birmingham B18 7QH, UK 